\title{
The effect of prenylamine on the QT interval of the resting electrocardiogram in patients with angina pectoris
}

\author{
D. OAKLEY \\ M.R.C.P. \\ R. PURITZ \\ M.R.C.P.
}

\author{
K. JENNINGS \\ M.R.C.P. \\ D. KRIKLER \\ F.R.C.P.
}

\author{
D. Chamberlain \\ F.R.C.P.
} The Division of Cardiovascular Medicine, Royal Postgraduate Medical School, Hammersmith Hospital,
London, and Cardiac Department, Royal Sussex County Hospital, Brighton

\begin{abstract}
Summary
Resting ECGs were recorded in 29 patients with angina pectoris before, during and after treatment with prenylamine $180 \mathrm{mg}$ daily. The QT interval became significantly prolonged after one week of treatment. The prolongation persisted as long as therapy was continued, which was up to 6 months. After withdrawal of treatment the QT interval returned to normal within 2 weeks. In this study no serious problems were encountered by those patients in whom the QT interval was prolonged.
\end{abstract}

\section{Introduction}

Prenylamine is a widely prescribed drug which is effective in reducing the number of attacks of angina pectoris and the frequency of glyceryl trinitrate consumption in patients with ischaemic heart disease. There is considerable variability in response from patient to patient (Tucker et al., 1974). Prenylamine has a number of different pharmacological actions including the depletion of adrenergic nerve endings of transmitter substance (Carlsson and Waldeck, 1968). It also possesses calcium inhibitory properties, but these are weak compared with more recent drugs such as verapamil and nifedipine (Fleckenstein, 1971).

Ventricular tachycardia has occurred in several patients taking prenylamine (Puritz et al., 1977; Bens et al., 1973; Jacovella and Fajola, 1976). In most cases the tachycardia has shown the characteristic rotating axis of torsade de pointes and the resting electrocardiogram (ECG) has shown QT interval prolongation. The incidence and degree of QT prolongation in patients taking prenylamine has not been systematically studied, nor is the time course of these changes known.
This study was designed to examine the relationship between prenylamine treatment and QT prolongation.

\section{Patients and methods}

Patients were selected from an out-patient clinic at the Royal Sussex County Hospital without age or sex restrictions. Apart from a characteristic history of angina pectoris, no other indicator of ischaemic heart disease was required. Any patient whose angina did not respond to $\beta$-adrenoceptor blocking drugs or in whom such drugs were contra-indicated was eligible for inclusion. Patients with serious impairment of hepatic or renal function, defects of cardiac conduction and uncompensated cardiac failure were excluded.

ECGs were recorded in the resting state using a Hewlett Packard 1500A electrocardiograph. After recording the baseline ECG patients were started on prenylamine $180 \mathrm{mg}$ daily. They were then reviewed at 1,3 and 6 weeks, after which they were treated with placebo tablets for a further 2 weeks before the final visit. In some cases, prenylamine was continued for 6 months before the 2-week placebo period. At each visit resting ECG, blood urea, electrolytes and calcium estimation, and tablet count were performed.

If at any stage the physician noted the QT interval to be markedly prolonged he could, at his discretion, withdraw the patient from the study. Under these circumstances a 24-hr ambulatory ECG tape was recorded before stopping the drug and starting the final 2 weeks' placebo period.

The ECGs were analysed by an independent observer at the Royal Postgraduate Medical School who was unaware of the sequence in which they had 
been recorded. The QT interval was measured from the earliest onset of electrical activity in the QRS complex to the end of the $T$ wave using the standard frontal plane leads. Where the end of the $T$ wave was obscured by tachycardia or prominent $U$ waves the downstroke of the $T$ wave was projected to the isoelectric line. The QT interval was corrected for heart rate using the formula $\mathrm{QT}_{\mathrm{c}}=\mathrm{QT} / \sqrt{ } \mathrm{RR}$ where $\mathrm{RR}=$ the mean of the 3 preceding cycle lengths in seconds.

Significant differences were sought using a 2-way analysis of variance.

\section{Results}

Twenty-nine patients, 18 male and 11 female, aged 30 to 73 years (mean 57) were entered into the trial. Two developed atrial fibrillation and in one the QT interval could not be measured in any lead. Of the remaining 26, 18 completed all the treatment periods. Reasons for failing to complete were as follows: one myocardial infarct, one coronary artery by-pass graft, one patient was intolerant of the drug, one patient did not tolerate withdrawal of the drug for the final placebo period and 3 were withdrawn by the physician for marked QT prolongation. All patients except one completed at least 3 weeks on prenylamine.

Of the 26 patients, 14 showed an increase in the QT interval of more than $0.04 \mathrm{sec}$ when taking prenylamine. Four showed lesser prolongation and 8 showed no change or shortening.

Figure 1 illustrates the data for the 19 patients who completed the trial. The numbers in brackets are the corresponding results for all the patients who completed each treatment period but subsequently withdrew for the reasons given. The percentage increase in $\mathrm{QT}_{\mathrm{c}}$ interval is plotted as a mean \pm the standard error. There was a significant lengthening of the $\mathrm{QT}_{\mathrm{c}}$ interval at one week compared with the control value $(P<0.001)$. There was a further lengthening at 6 weeks but this did not differ significantly from the one-week value. Fifteen patients persisted with prenylamine for 6 months but there was no further increase in the $\mathrm{QT}_{\mathrm{c}}$ interval. Two weeks after prenylamine withdrawal the $\mathrm{QT}_{\mathrm{c}}$ interval had dropped towards the control level. This was significantly lower than the 6-week level $(P<$ $0.001)$ and was not different from the baseline value. Figure 2 shows the distribution of the $\mathrm{QT}_{\mathrm{c}}$ intervals as a mean \pm one standard deviation for all 19 patients.

When all patients who completed each treatment period are considered the results are not materially altered. The numbers in brackets in Figs 1 and 2 refer to these patients and the levels of significance are unchanged.

\section{Electrolytes}

Serum potassium and calcium levels varied slightly in each patient throughout the study. In one case only was $\mathrm{QT}_{\mathrm{c}}$ prolongation from 0.42 to 0.45 associated with a drop in serum potassium fromo $3.72 \mathrm{mmol} / \mathrm{l}$ to $3.16 \mathrm{mmol} / \mathrm{l}$. After correction of the hypokalaemia, which was caused by steroid treatment, the $\mathrm{QT}_{\mathrm{c}}$ returned to $0 \cdot 43$. Exclusion of this patient from the analysis does not alter the levels of significance.

\section{Other drugs}

No patient started treatment with any drug known to be associated with QT prolongation during the period of the study. Most patients were taking other drugs but the doses of these were not altered

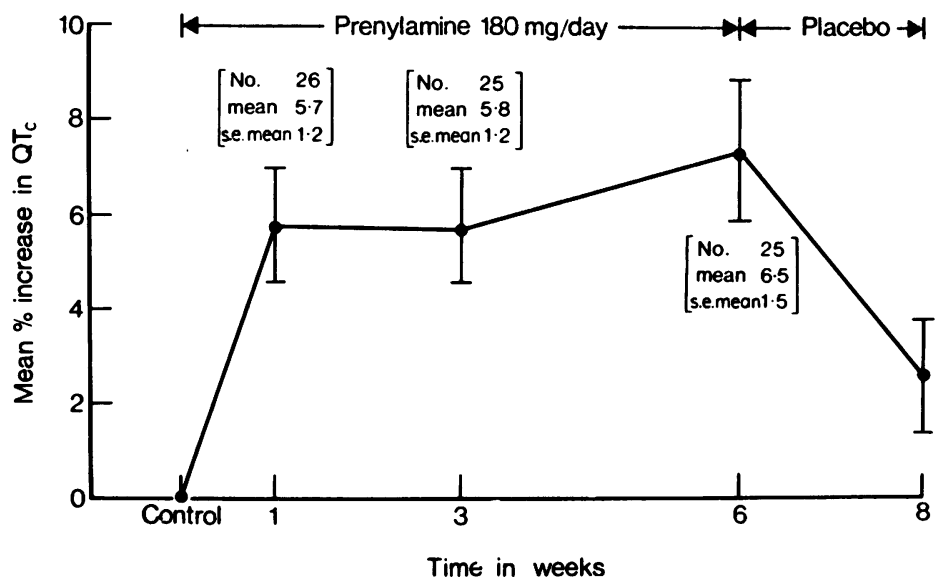

FIG. 1. Mean percentage increase in $\mathrm{QT}_{\mathrm{c}}$ interval \pm s.e. mean in 19 patients before, during and after prenylamine treatment. (Figures in brackets refer to all the patients who completed each treatment period.) 


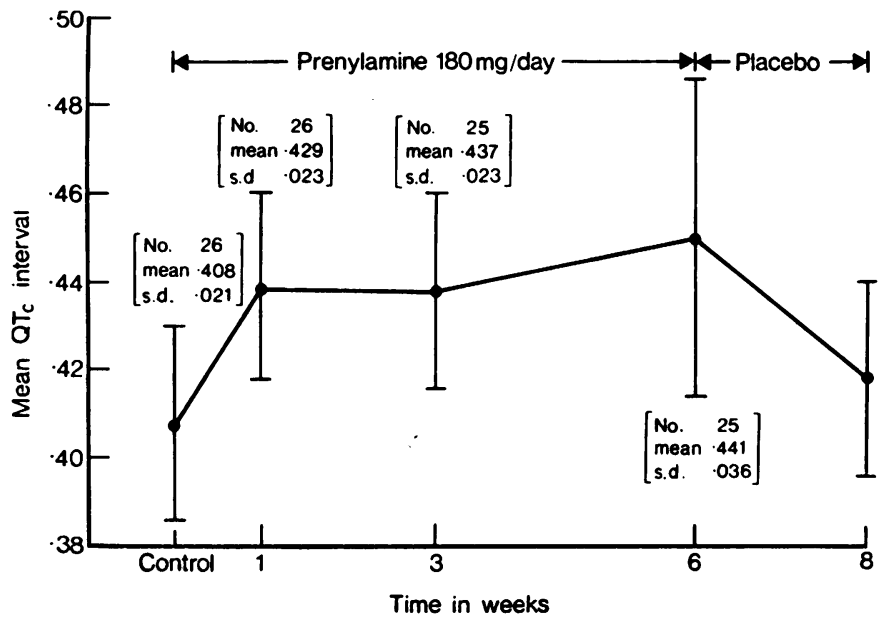

Fig. 2. Mean $Q T_{c}$ interval \pm one standard deviation in 19 patients before, during and after prenylamine treatment. (Figures in brackets refer to all the patients who completed each treatment period.)

immediately before or during the period of observation. These drugs were also continued in the same dose during the placebo period at the end of the study. The number of patients taking other drugs were as follows: tricyclic antidepressants, 3 ; digoxin, 4 ; thiazide or loop diuretics, $14 ; \beta$-blockers, 18 (propranolol 12, acebutolol 3, atenolol one, oxprenolol one, sotalol one). Figure 2 shows that the resting QT intervals before and after prenylamine treatment are within the normal range. The only factor which can be implicated in the lengthening of the QT interval is the introduction of prenylamine. An additive effect between prenylamine and another drug which the patient was taking cannot be excluded but is unlikely since QT prolongation was also observed in patients taking prenylamine only.

\section{Arrhythmias}

Three patients were monitored for $24 \mathrm{hr}$ before withdrawal of therapy. Two had occasional ventricular extrasystoles. One had frequent ventricular extrasystoles, occasionally occurring in pairs. No ventricular tachycardia was seen. None of the patients complained of palpitations or syncope.

\section{Discussion}

As might be expected from the sporadic case reports in the literature, this systematic study has shown that prenylamine significantly prolongs the $\mathrm{QT}_{c}$ interval but there is considerable variability from patient to patient. In this context it is worth noting that the metabolism and elimination of prenylamine is complex, involving several metabolites of uncertain pharmacological activity.
Prenylamine is also extensively bound to protein and lipoproteins (Dengler, Eichelbaum and Schomerus, 1968). One of the patients with marked hyperlipidaemia failed to show QT prolongation on prenylamine. In some subjects the QT interval did not lengthen, or the $T$ wave became flatter, making measurement of the interval less reliable. In other subjects the QT interval became unequivocally and markedly prolonged.

Prolongation of the QT interval is associated with dangerous ventricular arrhythmias. In the present patients $\mathrm{QT}_{\mathrm{c}}$ prolongation did not result in the manifestation of such arrhythmias but they were not monitored for prolonged periods. It does seem, however, that for a given degree of $\mathrm{QT}_{\mathrm{c}}$ prolongation there is great variability in the occurrence of arrhythmias in different patients. It is probable that other factors are involved. Perhaps those patients in whom ventricular repolarization is non-homogeneous, or variably affected by prenylamine owing to patchy ischaemia or fibrosis, are more at risk than those in whom repolarization is uniformly prolonged. Electrolyte changes may trigger arrhythmias in susceptible subjects.

The mechanism of QT prolongation by prenylamine is uncertain. Long QT intervals with ventricular tachycardia have been described in patients taking quinidine (Acierno and Gubner, 1951) and disopyramide (Meltzer et al., 1978), agents which fall into Class I of the Vaughan-Williams classification of anti-arrhythmic drugs. Other members of this class, e.g. imipramine, also cause QT prolongation. In contrast, Class IV agents, e.g. verapamil, do not affect the QT interval. The pharmacological actions 
of prenylamine on the cardiac cell action potential are less specific than these drugs. It has some Class IV activity (Fleckenstein, 1971) and also inhibits adrenergic influences on the heart by depleting nerve endings of transmitter substance (Carlsson and Waldeck, 1968). In this context, prenylamine may be considered to have Class II activity. Other Class II drugs, e.g. some $\beta$-adrenergic blocking agents, may cause QT lengthening on prolonged administration, but the extent and time course of the QT changes seen with prenylamine are quite different. Prenylamine does have Class I antiarrhythmic activity resulting in prolongation of the refractory period of atrial myocardium and in local anaesthetic properties (Lindner, 1963). It would be reasonable to postulate that this Class I effect on the action potential is responsible for the QT prolongation and that, in this respect, prenylamine is behaving no differently from quinidine.

The results of this study suggest that close electrocardiographic supervision of prenylamine therapy is essential, particularly in the early stages of treatment. Alternative therapy should be sought if the QT interval lengthens markedly and any symptoms suggesting arrhythmias should be thoroughly investigated. Since the drug has a prolonged elimination phase (Eichelbaum, Schomerus and Dengler, 1970) there is unlikely to be a rebound effect on angina following the prompt withdrawal. The $\mathrm{QT}_{\mathrm{c}}$ returns to normal within 2 weeks.

\section{Acknowledgment}

The Authors wish to thank Dr Ralph Rousell of Hoechst U.K. Limited who co-ordinated the initial stages of this study, provided placebo tablets and gave invaluable help throughout.

\section{References}

Acierno, L.J. \& Gubner, R. (1951) Utility and limitations of intravenous quinidine in arrhythmias. American Heart Journal, 41, 733.

Bens, J.L., Duboisset, M., Quiret, J.C., Lesbre, J.P. \& BernasConi, P. (1973) Syncopes par torsade de pointes induites ou favorisées par la prénylamine. Archives des maladies du coeur, des vaisseaux et du sang, 66, 1427.

Carlsson, A. \& Waldeck, B. (1968) On the mechanisms of action of prenylamine on tissue monoamines. In: Proceedings of the 2nd International Symposium on Prenylamine. Capri. Biochimica applicata, 14 (Suppl. 1), 41.

Dengler, H.J., Eichelbaum, M. \& Schomerus, M. (1968) Pharmacokinetics of prenylamine in man. In: Proceedings of the 2nd International Symposium on Prenylamine. Capri. Biochimica applicata, 14, (Suppl. 1), 26.

Eichelbaum, M., Schomerus, M. \& Dengler, H.J. (1970) Zur Pharmakokinetik von Prenylamin (Segontin) beim Menschen. Verhandlungen der deutschen Gesellschaft für innere Medizin, 76, 942.

Fleckenstein, A. (1971) Specific inhibitors and promotors of calcium action in the excitation-contraction coupling of heart muscle and their role in the prevention or production of myocardial lesions. In: Calcium and the Heart, pp. 135-188 (Ed by Harris, P. \& Opie, L.). Academic Press, London and New York.

Jacovella, G. \& Fajola, F.S. (1976) Effetti sfavorevoli dei farmaci: Sincope da prenilamina. Giornale italiano di cardiologia, 6, 547.

LINDNER, E. (1963) Studies of the antifibrillatory action of Segontin. Archives internationales de pharmacodynamie et de thérapie, 146, 485.

Meltzer, R.S., Robert, E.W., McMorrow, M. \& Martin? R.P. (1978) Atypical ventricular tachycardia as a manifestation of disopyramide toxicity. American Journal of Cardiology, 42, 1049.

Puritz, R., Henderson, M.A., Baker, S.N. \& Chamberlain, D.A. (1977) Ventricular arrhythmias caused by prenylamine. British Medical Journal, 2, 608.

Tucker, H., Carson, P., Bass, N. \& Massey, J. (1974) Prenylamine treatment in angina. British Heart Journal, 36, 1001. 